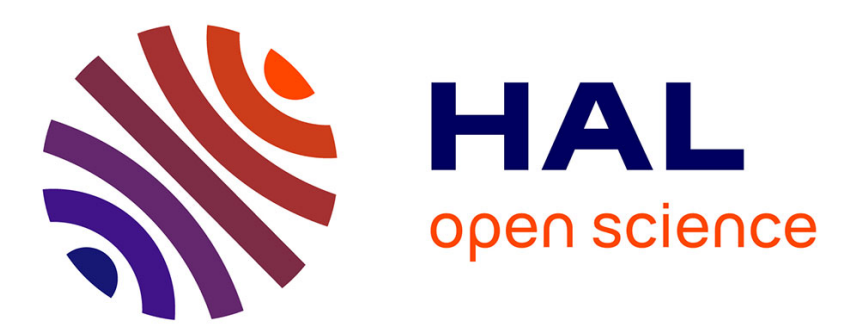

\title{
An original differentiation tool for identification on continuous structures
}

Catherine Chochol, Simon Chesné, Didier Rémond

\section{To cite this version:}

Catherine Chochol, Simon Chesné, Didier Rémond. An original differentiation tool for identification on continuous structures. Journal of Sound and Vibration, 2013, 332 (13), pp.3338-3350. 10.1016/j.jsv.2013.01.022 . hal-00807827

\section{HAL Id: hal-00807827 https://hal.science/hal-00807827}

Submitted on 11 Jun 2019

HAL is a multi-disciplinary open access archive for the deposit and dissemination of scientific research documents, whether they are published or not. The documents may come from teaching and research institutions in France or abroad, or from public or private research centers.
L'archive ouverte pluridisciplinaire HAL, est destinée au dépôt et à la diffusion de documents scientifiques de niveau recherche, publiés ou non, émanant des établissements d'enseignement et de recherche français ou étrangers, des laboratoires publics ou privés.

$$
\text { Copyright }
$$




\title{
An original differentiation tool for identification on continuous structures
}

\author{
Catherine CHOCHOL, Simon CHESNE, Didier REMOND \\ LaMCoS, INSA-Lyon, CNRS UMR5259, 18, rue des Sciences, F69621 Villeurbanne, \\ France
}

\begin{abstract}
The present contribution is dedicated to the identification of mechanical parameters, especially distributed parameters for continuous structures. In the following, the continuous time identification method is adapted and improved. Firstly an original expansion method using Gauss-points is presented. A novel differentiation method is then developed and compared to a classical one. These improvements are tested numerically and experimentally. This general method can be applied to system identification, structure identification, damage detection, etc. The main advantage of it is its high order differentiation capability. Therefore, this method is applied to structure monitoring on a cantilever beam in flexural motion (which involves a fourth derivative). In the treated example, the parameter $\rho S / E I$ and the fourth derivative $\partial^{4} v / \partial x^{4}$ are computed. The parameter $\rho S / E I$ is accurately reconstructed for the whole beam, using different sensor patch lengths and the identification method is shown to be a precise tool for damage location. The computed fourth derivative emphasises the discontinuity due to the crack. Using only the discontinuity property of a crack, no assumption is needed about crack behaviour. This novel differentiation technique makes it possible to compute changes and is of great interest for damage location.
\end{abstract}

Keywords: Chebyshev, continuous-time identification, numerical differentiation, partial derivation equation

\section{Introduction}

The identification of mechanical systems and structures is currently attracting much attention for monitoring and control purposes. In civil and Preprint submitted to Journal of Sound and Vibration

November 19, 2012 
aeronautical engineering, for example, optimizing structure life-time is crucial. Therefore robust monitoring is also vital for updating controllers. System control requires accurate models of the systems controlled, thus the formulation of the parameter identification procedures implemented is a critical step.

Various parametric identification methods [1](such as ARX, Box-Jenkins, etc.) have been developed over the last century. These methods seem difficult to implement in mechanical engineering, as the parameters identified have no mechanical meaning. Recently, D. Remond [2] proposed an improved continuous time identification method which permits the direct computation of mechanical quantities such as mass, damping, etc. This continuous-time identification method was applied on different multidegrees of freedom systems $[3,4,5]$ using different orthogonal bases (Chebyshev, Legendre, Fourier, etc.) and it has also been extended to non-linear systems [6]. All these studies give similar results.

In practice, many identification problems relating to mechanical vibration result in a further problem of derivative estimation. These estimation methods concern various domains and provide numerous applications. The usual methods use finite difference schemes, but often require a regularisation step, such as for force localisation [7, 8]. Some approaches comprise a natural regularisation dimension that involves using an integral formulation for boundary characterisation $[9,10,11]$, or polynomial approximation for damage detection [12]. D. Wu [12] adapted a part of this continuous time method for damage detection purposes on continuous structures. However, the whole continuous-time identification method has never been reformulated for a distributed parameter (continuous) structure, such as a beam or plate.

The purpose of this study was to adapt and improve the continuous time identification method proposed by D. Remond [2] for continuous structures. D. Remond [2] clearly separated this identification method into three steps: signal expansion, signal differentiation and parameter estimation. In this study, both expansion and differentiation steps are drastically improved. An original differentiation method is developed and adapted to partial differentiation. An example is then given of the application of this method for damage location on a beam structure.

In section 2 , the general formulation is presented briefly. The improvements developed are described and reformulated for continuous structures.

In section 3, this improved identification method is tested numerically on 
a cantilever beam. Two observations are discussed in order to understand the method's limits. The improved method is also compared to the classical one.

In section 4, this improved identification method is tested experimentally on a cantilever beam. The purpose of this experiment is to estimate the structure parameter $\rho S / E I$ and to reconstruct changes of this parameter. This physical parameter (proportional to the beam cross-section) is therefore identified as the damage indicator.

\section{Chebyshev polynomials, properties and a derivative formula- tion}

\subsection{Continuous Time identification method}

As presented in [2], the Continuous Time Identification method is usually based on three steps:

- Expansion step Firstly, the recorded signals are expanded on a truncated orthogonal basis. The choice of the orthogonal basis is not limited. The expanded signals are reduced to a few expansion coefficients.

$$
f(x) \approx \sum_{i=0}^{N} \lambda_{i} P_{i}(x)
$$

the recorded signal $f$ is expanded on orthogonal basis size $N . \lambda_{i}$ are the expansion coefficients. $P_{i}(x)$ is the ith orthogonal function of basis $P$.

- Differentiation step Secondly, the derivatives of the expanded signal are computed. For this step, the derivatives of the orthogonal functions are computed and derivatives of the signal are obtained from computed expansion coefficients.

$$
\begin{aligned}
f^{(z)}(x) & \approx \sum_{i=0}^{N} \lambda_{i}^{\{z\}} P_{i}(x) \\
\lambda_{i}^{\{z\}} & \approx \sum_{k=0}^{N} \alpha_{i+k}^{\{z\}} \lambda_{k}
\end{aligned}
$$

$f^{(z)}$ (the $z^{\text {th }}$ derivative of $f$ ) is expanded on the orthogonal basis $P$. $\lambda_{i}^{\{z\}}$ is the ith expansion coefficient of $f^{(z)}$. The expansion coefficients 
$\lambda_{i}^{\{z\}}$ are computed using the relationships between $P_{i}$ and its derivatives. These relationships are expressed by the $\alpha_{i+k}^{\{z\}}$ coefficients which can be computed using various methods. A novel method will be presented in section 2.2.2 and compared to a classical one in section 4 .

- Differential to algebraic equation transform - For this step, the differential equation governing the system behaviour is used. All the derivatives in the differential equation are replaced by their expansion (computed in the differential step). The result of this step is an algebraic equation composed of expansion coefficient arrays and parameters. The parameters are computed using this algebraic equation.

The following is an example. Let the linear differential equation be:

$$
f^{(4)}(x)+A_{3} f^{(3)}(x)+A_{2} f^{(2)}(x)+A_{1} f^{(1)}(x)+A_{0} f(x)=0
$$

This differential equation is composed of the derivatives of $f$ and the $A_{j}, j=0 . .3$ parameters and is transformed into the following algebraic equation:

$$
\lambda_{i}^{\{4\}}+A_{3} \lambda_{i}^{\{3\}}+A_{2} \lambda_{i}^{\{2\}}+A_{1} \lambda_{i}^{\{1\}}+A_{0} \lambda_{i} \approx 0
$$

Using this set of algebraic equations (for $i=0 . . N$ ), the $A_{j}$ parameters can be computed using a simple least square method.

\subsection{Chebyshev polynomials properties and novel derivative formulation}

\subsubsection{Expansion with Gauss-points}

The aim of the expansion with Gauss-points is to optimize the expansion step. The $\lambda_{i}$ expansion coefficients are expressed as a scalar product:

$$
\lambda_{i}=<f(x), P_{i}>=\int_{\Omega} f(x) w(x) P_{i}(x) \mathrm{d} x
$$

where $\Omega$ is the domain of orthogonality. $w(x)$ is a weighting function associated with the basis $P$.

Traditionally, the trapezoidal rule is used to compute the integral and the $\lambda_{i}$ coefficients. This approach is accurate only if $f$ is recorded for a large number of samples, or if $f$ and $P_{i}$ vary slowly in the interval. 
An interesting property of Chebyshev orthogonal functions is their exact discrete scalar product [13]. This scalar product can be transformed into:

$$
\lambda_{i}=\sum_{j=1}^{N+1} f\left(x_{j}\right) P_{i}\left(x_{j}\right)
$$

where $N+1>i$ and $x_{j}$ for $j=1 . . N+1$ are the zeros (or Gauss-points) of Chebyshev function $P_{N+1}$ of order $N+1$. If $f$ is recorded at all $x_{j}$ points, using (6), the $\lambda_{i}$ can be computed precisely with the discrete and finite formulation.

This is true only if $f$ can be expanded on the basis of size $N(f(x)=$ $\left.\sum_{i=0}^{N} \lambda_{i} P_{i}(x)\right)$. In the case of the truncated expansion(1), the effect of the approximation is negligible in comparison to the effect of the trapezoidal rule, with the same number of samples. This comparison will be shown in section 3.2.2.

\subsubsection{Differentiation}

A key challenge of this study was to optimize the differentiation step. In order to calculate the derivatives of $f$, In [2], the author uses a derivative matrix $[D]$. This $[D]$ matrix is composed of the $\alpha_{i+k}^{\{z\}}$ coefficients expressed previously (see eq.(2)). This method consists in assuming that the derivative of $f$ is equal to a combination of the derivatives of $P_{i}$. However, this simplification is not optimal. As explained by D-Y Lui [14], the estimation of the derivative is corrupted by bias term errors that originate from the truncation of the expansion of $f$. The aim of our novel differentiation tool is to eliminate the bias term by integrating the expression of the scalar product, as proposed by M. Mboup [11].

This novel differentiation method is used to compute the expansion of $\left[f^{(z)} \cdot u_{\gamma}\right]$ (function $u_{\gamma}$ will be explained in the next section). It is obtained via a two-step process. Firstly, the scalar product of $\left[f^{(z)} \cdot u_{\gamma}\right]$ by $P_{i}$ is integrated by parts. Secondly, it is rewritten as a combination of scalar products of $f$ by $P_{k}$, weighted by a test function $u_{\gamma-z}$.

If we write the expansion of $f^{(z)}$, the $z$-order derivative of $f$, in the integral form (5), the expression can be integrated by parts in order to obtain (see Appendix A for proof):

$$
\begin{aligned}
\tilde{\lambda}_{i}^{\{z\}} & =<f^{(z)} \cdot u_{\gamma}, P_{i}> \\
& =(-1)^{z} \int_{-1}^{1} f(x)\left[u_{\gamma}(x) w(x) P_{i}(x)\right]^{(z)} \mathrm{d} x
\end{aligned}
$$


where $u_{\gamma}$ is chosen such that the integrated parts are equal to zero, $i$ is the order of the Chebyshev function and $\{z\}$ is the differentiation order. $\tilde{\lambda}_{i}^{\{z\}}$ denotes the expansion coefficient of $f^{(z)} \cdot u_{\gamma}$ (unlike $\lambda_{i}^{(z)}$ which is the expansion coefficient of $\left.f^{(z)}\right)$. For the following, we can simply choose:

$$
u_{\gamma}(x)=\left(1-x^{2}\right)^{\gamma} \quad \gamma \geq z+1 / 2
$$

By analytic calculation of $\left[u_{\gamma}(x) w(x) P_{i}(x)\right]^{(z)}, \tilde{\lambda}_{i}^{\{z\}}$ can be written as a combination of expansion coefficients of $f \cdot u_{\gamma-z}$, with $u_{\gamma-z}(x)=\left(1-x^{2}\right)^{\gamma-z}$.

$$
\tilde{\lambda}_{i}^{(z)}=\sum_{k=-z}^{z} \alpha_{i+k}^{(\gamma, z)}<u_{\gamma-z} \cdot f, P_{i+k}>
$$

$\alpha_{i+k}^{(\gamma, z)}$ coefficients depend on the $z, i, \gamma$ and $k$. For $u_{\gamma}$ chosen before, $\alpha_{i+k}^{(\gamma, z)}$ values are given up to the fourth derivatives in Appendix B.

This novel differentiation method is bias-free, contrary to the operator $[D]$ and it is also computable using other orthogonal bases. The improvements of this differentiation method are shown in section 3.2.3.

\subsubsection{Reformulation for continuous structures}

\section{Expansion.}

With a continuous structure, the signal often depends on more than one variable. For example, for a beam, the displacement field depends on time and space (along the beam axis).

Now let us consider a signal recorded along two different dimensions/directions $\chi_{1}$ and $\chi_{2}$. This signal can be expanded through both domains by writing:

$$
f\left(\chi_{1}, \chi_{2}\right) \simeq \sum_{i_{1}=1}^{N} \sum_{i_{2}=1}^{M} \lambda_{i_{1}, i_{2}} P_{i_{1}}\left(\chi_{1}\right) P_{i_{1}}\left(\chi_{2}\right)
$$

where $N, M$ are the sizes chosen for the orthogonal basis of $\chi_{1}$ and $\chi_{2}$ respectively. In order to calculate the $\lambda_{i_{1}, i_{2}}$ coefficients, $\chi_{1}$ and $\chi_{2}$ samples will be the zeros of $P_{N+1}$ and $P_{M+1}$.

$f$ recorded at the sample points and $P$ computed at the same points can be transformed into matrices in the same way as that developed in [2]. The $\lambda_{i_{1}, i_{2}}$ are then obtained by writing:

$$
\boldsymbol{\lambda}_{N, M}=\boldsymbol{P}\left(\chi_{\mathbf{1}}\right)_{N, N+1}^{\dagger} \mathbf{f}_{N+1, M+1} \boldsymbol{P}\left(\chi_{\mathbf{2}}\right)_{M+1, M}^{\dagger}
$$


where $\cdot_{A, B}^{\dagger}$ is the pseudo-inverse of $\cdot A, B$, which is a A-by-B matrix and with

$$
\begin{aligned}
& \mathbf{f}=\left[\begin{array}{ccc}
f\left(\chi_{1,1}, \chi_{2,1}\right) & \cdots & f\left(\chi_{1, N+1}, \chi_{2,1}\right) \\
\vdots & & \ddots \\
f\left(\chi_{1,1}, \chi_{2, M+1}\right) & \cdots & f\left(\chi_{1, N+1}, \chi_{2, M+1}\right)
\end{array}\right] \\
& \boldsymbol{P}\left(\chi_{\mathbf{1}}\right)= {\left[\begin{array}{ccc}
P_{0}\left(\chi_{1,1}\right) & \cdots & P_{N}\left(\chi_{1,1}\right) \\
\vdots & \ddots & \vdots \\
P_{0}\left(\chi_{1, N+1}\right) & \cdots & P_{N}\left(\chi_{1, N+1}\right)
\end{array}\right] } \\
& \boldsymbol{P}\left(\chi_{\mathbf{2}}\right)=\left[\begin{array}{ccc}
P_{0}\left(\chi_{2,1}\right) & \cdots & P_{M}\left(\chi_{2,1}\right) \\
\vdots & \ddots & \vdots \\
P_{0}\left(\chi_{2, M+1}\right) & \cdots & P_{M}\left(\chi_{2, M+1}\right)
\end{array}\right]
\end{aligned}
$$

Partial differentiation.

In (9), the derivatives of $f \cdot u$ are computed using the scalar product of the signal $f$ multiplied by $u_{\gamma-z}$. This formulation can be extended to a two dimension case. Let us consider the $z$-order partial derivative of $f$, with respect to the direction $\chi_{d}(d=1$ or $d=2)$ :

$$
{ }_{d} \tilde{\lambda}_{i}^{(z)}=\sum_{k=-z}^{z} \alpha_{i+k}^{\left(\gamma_{d}, z\right)}<u_{\gamma_{d}-z}\left(\chi_{d}\right) \cdot f, P_{i+k}>
$$

the ${ }_{d} \tilde{\lambda}_{i}^{(z)}$ are then rearranged in a matrix called ${ }_{d} \tilde{\lambda}^{(z)}$.

From partial derivative to algebraic equation.

Let us consider a partial differential equation of $f, f$ depending on two variables $\chi_{1}, \chi_{2}$ :

$$
\sum_{z=0}^{Z}\left[A_{1, z} \frac{\partial^{z} f}{\partial \chi_{1}^{z}}+A_{2, z} \frac{\partial^{z} f}{\partial \chi_{2}^{z}}+A_{1,2, z} \frac{\partial^{2 z} f}{\partial \chi_{1}^{z} \partial \chi_{2}^{z}}\right]=G
$$

where $A_{1, z}, A_{2, z}, A_{1,2, z}$ are constants (which depends on the physics studied), $G$ is a function of $\chi_{1}$ and $\chi_{2}$. It has been shown that a function $u_{\gamma}$ is needed to calculate the partial derivatives of $f$. However, in equation (14), different orders of differentiation are involved. Therefore the only way to make use of the previous differentiation process is to select $\gamma$ for the highest order $Z$ $(\gamma \geq 1 / 2+Z)$. Indeed, (14) can be written as:

$$
U \sum_{z=0}^{Z}\left[A_{1, z} \frac{\partial^{z} f}{\partial \chi_{1}^{z}}+A_{2, z} \frac{\partial^{z} f}{\partial \chi_{2}^{z}}+A_{1,2, z} \frac{\partial^{2 z} f}{\partial \chi_{1}^{z} \partial \chi_{2}^{z}}\right]=U \cdot G
$$


with $U=u_{\gamma_{1}}\left(\chi_{1}\right) \cdot u_{\gamma_{2}}\left(\chi_{2}\right)=\left(1-\chi_{1}^{2}\right)^{\gamma_{1}}\left(1-\chi_{2}^{2}\right)^{\gamma_{2}} \cdot \gamma_{1}$ and $\gamma_{2}$ can be freely chosen by the user.

Then,(15) is reduced to an algebraic equation:

$$
\sum_{i=0}^{Z}\left(A_{1, z \mathbf{1}} \tilde{\boldsymbol{\lambda}}^{(z)}+A_{2, z \mathbf{2}} \tilde{\boldsymbol{\lambda}}^{(z)}+A_{1,2, z 1,2} \tilde{\boldsymbol{\lambda}}^{(2 z)}\right)=\tilde{\boldsymbol{\lambda}}_{G}
$$

${ }_{1} \tilde{\lambda}^{(z)},{ }_{2} \tilde{\lambda}^{(z)}$ and ${ }_{1,2} \tilde{\lambda}^{(2 z)}$ are the matrices computed using the process summarized in Fig. 1. $\tilde{\lambda}_{G}$ is the expansion matrix of $G \cdot U$.

All $A_{1, z}, A_{2, z}, A_{1,2, z}$ constants can be determined with the set of equations generated by $(16)$.

Figure 1: Process for computation of $\left.\left[\tilde{\lambda}_{1}^{(z)}\right],{ }_{2} \tilde{\lambda}^{(z)}\right]$ and $\left[{ }_{1,2} \tilde{\lambda}^{(2 z)}\right]$ matrices (blue: recorded, green: computed, pink: expansion coefficients). $p=1$ or $p=2, p *=1$ or $p *=2$ with $p * \neq p$

\section{Numerical simulation}

\subsection{Computation framework}

The vibration analysis of the cantilever beam is used as an example to demonstrate the improvements of the method proposed and determine its limits. Indeed, the computation of the fourth derivative of the EulerBernouilli beam equation is a good challenge for this novel identification method. Furthermore the cantilever beam response consists in propagating and evanescent waves. Evanescent waves emanates from the discontinuities at the ends and can be written in the exponential form. These waves are usually hard to expand. Finally, the displacement field obtained with a cantilever beam is not symmetric. It becomes therefore interesting to expand it on a basis made of symmetric functions.

The forced response of this free-clamped beam is computed analytically. The dimensions of the beam are given in Fig. 2 and are similar to those of the beam studied experimentally in section 4. For section 3.3.2, a numerical model of a crack and a thickness reduction of $10 \%$ on $0.5 \%$ of the beam length are considered. The forced response of the cracked beam is also computed analytically.

Additive and multiplicative noise is added to the beam response in order 
Figure 2: Cantilever beam dimensions and model

to simulate sensors and amplifiers noise. For each numerical application, the process is tested on a Monte Carlo simulation with 1000 runs. The distributed displacement is computed as follows:

$$
v^{\text {noisy }}(x, t)=v^{\text {exact }}(x, t) \Delta v_{m} e^{j \Delta \phi}+\Delta v_{a}
$$

$\Delta v_{m}$ is a real Gaussian number with a mean value equal to unity and a standard deviation equal to a chosen percentage of the magnitude of the displacement, $\Delta \phi$ is another Gaussian random real number of a null mean value and a standard deviation of $1 \mathrm{deg}$ and $\Delta v_{a}$ is a real Gaussian number of a null mean value and a standard deviation equal to a chosen magnitude percentage of the displacement.

\subsection{Application to beam test case}

\subsubsection{Formulation}

The simple 3 steps process presented in 2.1 will be applied the the cantilever beam identification. The equation of a Euler-Bernoulli beam defining the behaviour of a bending beam without external excitation can be expressed as the sum of partial derivatives of the displacement $v$, with respect to time $t$ and position $x$ :

$$
\frac{\partial^{4} v}{\partial^{4} x}(x, t)=\frac{\rho S}{E I} \frac{\partial^{2} v}{\partial^{2} t}(x, t)
$$

where $\rho$ is the density, $E$ the Young modulus of the material, $S$ the crosssection area and $I$ the flexural inertia of the beam. With a crack, the cross-section area $S$ and flexural inertia $I$ will change locally. This change will be reconstructed by the identification method proposed. The aim of this application is to evaluate $\frac{\rho S}{E I}$.

Multiplying (18) by $U(x, t)=\left(1-x^{2}\right)^{5 / 2}\left(1-t^{2}\right)^{3 / 2}$, the following algebraic equation is deduced:

$$
{ }_{x} \tilde{\lambda}^{(4)}=\frac{\rho S}{E I} t^{t} \tilde{\lambda}^{(2)}
$$

The space and time derivatives are reconstructed through this identification process. The matrices ${ }_{x} \tilde{\lambda}^{(4)}$ and ${ }_{t} \tilde{\lambda}^{(2)}$ are calculated as presented in Fig. 1 . 


\subsubsection{Signal expansion improvements}

The expansion using Gauss-points is compared to the expansion process using the trapezoidal rule. The methods proposed for one and twodimensional cases are similar, therefore the harmonic response of the healthy beam (depending only on $x$ ) is expanded. Indeed, the expansion results are easier to interpret for a one-dimensional case. The expansion of the signal (contrary to the signal itself) is studied, as method errors such as bias effect are more visible on the expansion coefficients.

A reference expansion is computed. To perform this reference expansion, the beam response is computed at 1000 Gauss-points. This expansion can be considered as a reference, since the difference between a 1000 Gausspoint expansion and a 2000 Gauss-point expansion is smaller than -10dB. The error in $\mathrm{dB}$ is computed as follows:

$$
\operatorname{error}(\mathbf{d B})=\log _{10}\left|\frac{\boldsymbol{\lambda}_{i C}-\boldsymbol{\lambda}_{i_{r e f}}}{\boldsymbol{\lambda}_{\text {iref }}}\right|
$$

$\boldsymbol{\lambda}_{\boldsymbol{i} \text { ref }}$ being the reference expansion coefficient, and $\boldsymbol{\lambda}_{\boldsymbol{i} C}$ the computed coefficient. For the results concerning the second mode shown in Fig. 3, only

Figure 3: Error $(\mathrm{dB})$ on the expansion coefficient for the second mode of the beam. (a): noise-free case, (b): with $15 \%$ noise. The results are given for order $i<6$ (for $i>6$, the expansion coefficients are $10^{3}$ smaller than the first 6 ). The grey lines represent the dispersion on the expansion error (obtained with 1000 runs on the Monte Carlo application)

the first 6 expansion coefficients are relevant and presented. For the noisefree case (left), the expansion error is drastically reduced using Gauss-points (from $-1 \mathbf{d B}$ to $-4 \mathbf{d B}$ on average).

With $15 \%$ noise, the expansion based on only a few sensors (in this example 24 or 31) is very sensitive to noise. Therefore the expansion results are averaged over 10 runs. This averaged process is tested on a Monte Carlo application with 1000 runs.

Even with noise, the expansion error is smaller with Gauss-points than when using the trapezoidal rule. The improvement is smaller than for the case without noise, but error dispersion is greater when using the trapezoidal rule. With 24 linear-spaced samples, the maximum error is almost always greater than $0 \mathbf{d B}$ (except for $i=0$ ). With Gauss-points the maximum 
expansion error is close to the mean error, therefore limiting the risk of inaccurate expansion.

\subsubsection{Differentiation improvements}

Differentiations using the $\mathbf{D}$ matrix proposed in [2] and using the novel method explained in section 2.2.2 are compared. The harmonic response of the healthy beam (depending only on $x$ ) studied previously is differentiated. In order to apply these observations to the experimental settings, the fourth derivative is computed. The expansion of the fourth derivative calculated analytically and expanded with 1000 Gauss points is considered as a reference. In Fig. 4, the error on computation of the fourth derivative

Figure 4: Error $(\mathrm{dB})$ on the fourth-order differentiation coefficient for the beam second mode. (a): noise-free case, (b): with $15 \%$ noise. The results are given for the order $i<6$ (for $i>6$, the expansion coefficients are $10^{3}$ smaller than for the first 6 ). The grey lines represent the dispersion on the expansion error (performed with 1000 runs)

with a truncated expansion $(N=27$ and $N=28)$ is presented. These two truncation orders are selected for the signal studied (second modal response). Indeed, the $28^{\text {th }}$ expansion coefficient is smaller than the first 27 coefficients. This basis extension clearly reveals the bias effect.

For the noise free-case, considerable improvement $(-10 \mathbf{d B})$ is achieved with the novel differentiation method. The bias effect with the operator $[D]$ appears on high order coefficients $(>3)$. With noise, the bias effect is amplified, as shown in Fig. 4. Indeed, both methods give similar results for a sufficiently truncated basis $(N=27)$. However, the method based on the operator $\mathbf{D}$ is very sensitive to the truncation order $N$. For $N=28$, the high order coefficients are corrupted by the bias and the error is greater than 1dB. This error induces extra oscillations on the computed derivative, as shown in Fig. 5. With the novel differentiation method, the error on high order coefficients is significantly reduced. It still adds extra-oscillations but their amplitude is reduced compared to the $\mathbf{D}$ operator method. Therefore truncation order $N$ does not require precise tuning.

\subsection{Limits of the method proposed}

3.3.1. On the link between the number of samples and the wave number

In Fig. 3, the expansion precision is clearly related to the number of samples (Gauss-points) used for the computation. In (6), $N+1$ Gauss- 
Figure 5: displacement $v \cdot u$ (a) and fourth derivative $v^{(4)} \cdot u$ (b) for the cantilever beam. Light grey: signal computed analytically; light pink: computed with the novel differentiation method; dark purple: computed with the $[D]$ operator

points are needed for the computation of $N$ expansion coefficients. In this section, the link between the truncation order $N$ and the composition of the signal frequency are studied. For non-dimensional considerations, the signal frequency composition is replaced by the wave number. The latter does not depend on the structure's length or material.

For this analysis we define the identification precision $\Gamma$ as:

$$
\Gamma(\mathbf{d B})=\log _{10}\left|\frac{\frac{\rho \boldsymbol{S}}{\boldsymbol{E}}_{I D}-\frac{\rho \boldsymbol{S}}{\boldsymbol{E} \boldsymbol{I}} T H}{\frac{\boldsymbol{\rho} \boldsymbol{S}}{\boldsymbol{E} \boldsymbol{I}}_{T H}}\right|
$$

where $\cdot_{I D}$ is the parameter identified and $\cdot_{T H}$ is the theoretical value. For example if $\Gamma=-2$, then the parameter is accurate with $1 \%$ error.

in Fig. 6, the precision on the parameter identified is presented for the wave number $k$ between 0.1 and 3 . For the noise free case, precision is clearly related to the number of samples. For $N=14$, for $k>1$, the error increases fast. Indeed, a sufficiently high number of polynomials is needed to accurately expand the signal. These noise-free observations set the upper limit of $k$, regarding to $N$. The performances of the method

Figure 6: Identification precision (dB) for the cantilever beam. (a): noise-free case; (b): with $5 \%$ noise. Averaged precision obtained with 1000 runs for the noisy case. Results for the different truncation order $N$

proposed are evaluated by a Monte Carlo simulation with 100 runs of this averaged process. The performance of the identification is poor for small wave number $(k \leq 0.5)$. Indeed, for this study the expansion matrices are of size $N$-by- $N$. These matrices are almost empty for $k \leq 0.5$, as the signal is only expanded on the first Chebyshev functions. Indeed, the signal oscillates slowly and is therefore expanded only on the few first orthogonal functions. Therefore, even if the coefficients are averaged, they are very sensitive to noise. These observations on the noisy case set the lower limit 
of $k$, regarding $N$.

In order to obtain maximum advantage from this identification method, it is crucial to select the number of samples $N$ for a given wave number $k$.

\subsubsection{On the link between the damage position and the Gauss point location}

For this section, we fix $N=16$ (the same as for the experimental results). In order to satisfy the previous considerations, we choose $k=1$. A crack ( $10 \%$ height, $0.5 \%$ length) is moved numerically from one end to the other of the beam considered. The signal is expanded on the Chebyshev basis for each crack position. The computed expansion coefficients ${ }_{x} \tilde{\lambda}_{i}^{(4)}$ of the cracked beam and the healthy beam are compared. In Fig. 7, the results are presented for $i=2,3,6$ and 10 (the observations are similar for other $i$ ). In Fig. 7, the difference between the cracked and healthy expan-

Figure 7: Computed expansion coefficients of the fourth derivative with respect to space. Comparison between cracked and healthy expansion. From (a) to (d): expansion order $i=2,3,6$ and 10 . The grey vertical lines correspond to the Gauss points of the Chebyshev polynom of order $i$.

sion coefficients is clearly sensitive to the position of the crack regarding to the Gauss-points. Indeed a crack located near the Gauss-points of the ith polynom implies a noticeable change on the computed expansion coefficient of order $i$.

Therefore the crack can be located depending on which expansion coefficients change.

\subsection{Summary of numerical considerations}

It has been shown previously that the identification results depend on the following considerations:

- for a truncation order $N, N+1$ Gauss-points are required;

- the novel differentiation method is less sensitive to the truncation order $N$;

- a sufficiently high truncation order $N$ is required in order to compute the signal expansion precisely (refer to Fig. 6, noise free case); 
- a sufficiently high wave number $k$ is required in order to separate signal expansion and noise expansion accurately (refer to Fig. 6, noisy case);

- crack position can be computed depending on which expansion coefficient is affected (refer to Fig. 7).

\section{Experimental results}

\subsection{Implementation setup}

The novel differentiation formulation reformulated for continuous structures is applied to a cantilever beam, although the experimental technique can be applied to any type of structure. The parameter $\rho S / E I$ and the fourth derivative of the displacement(with respect to space) are computed. The displacement of this beam is reconstructed with selected time samples and space positions using a laser vibrometer (PSV400). Here, we study the forced response of this cantilever beam. $1.1 \mathrm{~m}$ long in which a crack is imposed (a notch $3 \mathrm{~mm}$ in width and $2.5 \mathrm{~mm}$ in depth). For this experimentation, a relatively large crack is chosen as a first test of this method. Fig. 8 shows the beam, the imposed crack and laser measurements at different positions. A sensor array provides 16 measurements at 16 different sample positions (as shown in Fig. 8). For this experiment, the limits of the method

Figure 8: Experimental implementation: cantilever beam, imposed crack and laser measurements at different sample positions (for one sensor array)

considered (presented in section 3.3) were taken into account. Each sensor array has 16 sample positions. Therefore, the signal is expanded on the basis of 15 Chebyshev functions. In order to ensure a minimal wave number equal to 1, the length of the sensor array is adapted at each excitation frequency. For example, at the $5^{\text {th }}$ flexural mode of the beam, the sensor array length is equal to $1 / 5^{\text {th }}$ of the beam length. No specific hypothesis can be made regarding the crack location on the sensor array. Therefore the sensor array is shifted along the beam. If the crack were located in a dead zone for a given sensor array position, it would be located in a sensitive zone for the next sensor array position, as shown in Fig. 9. The experimental application of this method was tested for different excitation frequencies. Each experiment (for a given sensor array position and for a given excitation 
Figure 9: Experimental implementation: cantilever beam, imposed crack and laser measurements at different sample positions (for one sensor array)

frequency) was repeated twice. As the results for the different excitation frequencies were similar, the dispersion of the results for a single frequency is presented in the next section.

\subsection{Results and discussion}

Parameter $\rho S / E I$ and the fourth derivative $\left(\partial^{4} v / \partial t^{4} \cdot u\right)$ can be evaluated for all the sensor array locations for undamaged $(x<0.8 m$ and $x>0.8 m))$ and damaged cases $(x \approx 0.8 m)$.

This identification method has two main applications. The first is linked to the material properties of the structure. It permits the identification of a global $\rho S / E I$ for the whole beam. This method has many applications, such as model updating for a controller and monitoring of Young's Modulus (material aging). The second involves more geometrical properties of the structure. It permits the computation of the dispersion of $\rho S / E I$ along the beam for each sensor array.

Fig. 10 (left) shows the ratio between the identified and theoretical $\rho S / E I$. The value of the mean identified parameter $\rho S / E I$ is computed and plotted in dashed lines: dark grey for $k \approx 1$ and light grey $k \approx 0.8$. For both cases, the mean value is close to the theoretical one ( ratio equal to 1.16 for $k=0.8$ and 0.85 for $k=1$ ). The mean value can be corrupted by noise which could explain the dispersion of the measurements. The theoretical value is also roughly estimated with the beam dimensions and the properties of the steel. The dispersion of $\rho S / E I$ is plotted in Fig. 10 (left). Each filled box corresponds to the parameter dispersion at a single sensor array position. The computed parameter values oscillate between $1 / 2$ and 3 times the mean value, except when the sensor array is located near $x=0.8 \mathrm{~m}$. At this location, the computed parameter becomes negative or is greater than 4 times the mean value. The oscillating values (between $1 / 2$ and 3 times the mean value) can be explained by the variability due to the noise. The assumption of a continuous structure is not accurate at the damage location, therefore the computed parameter can be negative. Conversely, a negative parameter shows a breach in the formulated hypothesis and therefore damage. The effect of damage (or a discontinuity) is similar to that of noise. Indeed, a 
discontinuity changes the slope of the signal slope locally. This local change drastically increases the high order coefficient of expansion. In the case of noise, the effect of these high order coefficients is smoothed by the computation of a mean value. In the case of damage, the effect of these high order coefficients cannot be smoothed by the same technique. Therefore the value of the computed parameter at damage location can increased by more than 4 times, due to these high order coefficients.

This reasoning is confirmed in Fig. 10 (right). Indeed the calculation of the displacement's fourth derivative with respect to space emphasises these high order coefficients. Near the damage location, the computed fourth derivative consists of high-oscillating terms. For both cases $(k \approx 0.8$ and $k \approx 1)$, the maximum value of the fourth derivative is located at the Gauss-point closest to the damage. These results demonstrate an alternative method

Figure 10: $\boldsymbol{\rho} \boldsymbol{S} / \boldsymbol{E} \boldsymbol{I}_{I D} / \boldsymbol{\rho} \boldsymbol{S} / \boldsymbol{E} \boldsymbol{I}_{T H}$ (a) and $\left(\partial^{4} v / \partial x^{4} \cdot u\right)(\mathrm{b})$ for the cantilever beam, results based on a experimental data (excitation frequency equal to $1471 \mathrm{~Hz}$ ). On the left, the light and dark grey dashed lines correspond to the mean values ( for $k \approx 0.8$ and $k \approx 1$ respectively)

for damage location. Indeed, with a restricted number of samples (here 16 samples), it was shown that $\rho S / E I$ and the fourth derivative $\partial^{4} v / \partial x^{4}$ can be computed. The parameters studied are very sensitive to damage (a discontinuity). $\rho S / E I$ becomes negative when the continuity assumption is no longer valid. The damage can also drastically increase the $\rho S / E I$ value. The method proposed is capable of computing these changes and thus locating the damage accurately.

\section{Conclusion}

In this contribution, a novel differentiation technique was reformulated for partial differentiation. Using signal expansion and our novel differentiation technique, the partial differential equation governing system behaviour was transformed into an algebraic equation. This novel differentiation technique is bias-free and therefore less sensitive to the basis truncation order. A basis size extension leads to increasing the precision of identification, contrary to the operational matrix traditionally computed for this application. No assumption about boundary conditions is made. Therefore this identification technique can be used with unknown boundary conditions. 
The method was applied to a cantilever beam to locate imposed damage, although the experimental technique can be applied to any type of structure. Indeed, parameter $\rho S / E I$ and the fourth derivative $\partial^{4} v / \partial x^{4}$ were computed, using a signal recorded experimentally. Parameter $\rho S / E I$ was accurately reconstructed for the whole beam, using different sensor patch lengths. The damage was accurately located using this identification method. The computed fourth derivative emphasised the discontinuity due to the crack. These changes in continuity could be computed with the novel differentiation technique, making it very interesting for damage location. This technique shows also good capabilities for the estimation of material properties such as the Young Modulus. Therefore, this technique could be applied to material ageing estimation.

Further research should extend this technique to structure response with white noise excitation. Then, for example, this method could be applied to the ambient response of a bridge. The link between crack size, noise level and method regularization is also under investigation.

\section{Appendix A. Proof of (7)}

The scalar product between $P_{i}$ and $u_{\gamma} \times f^{(z)}$ is defined as :

$$
\tilde{\lambda}_{i}^{\{z\}}=\int_{-1}^{1} f^{(z)}(x)\left[u_{\gamma}(x) w(x) P_{i}(x)\right] \mathrm{d} x
$$

Integrating once by part:

$$
\begin{aligned}
\tilde{\lambda}_{i}^{\{z\}} & =\left[f(x)^{(z-1)}\left[u_{\gamma}(x) w(x) P_{i}(x)\right]\right]_{-1}^{1}-\int_{-1}^{1} f(x)^{(z-1)}\left[u_{\gamma}(x) w(x) P_{i}(x)\right]^{(1)} \mathrm{d} x \\
& =-\int_{-1}^{1} f(x)^{(z-1)}\left[u_{\gamma}(x) w(x) P_{i}(x)\right]^{(1)} \mathrm{d} x
\end{aligned}
$$

This is true if $u_{\gamma}(1)=u_{\gamma}(-1)=0$ and $f(1)^{(z-1)}, f(-1)^{(z-1)}, w(1), w(-1), P_{i}(1)$ and $P_{i}(-1)$ finite. This assumption is true for $w$ and $P_{i}$ and most of the 
time true for $f^{(z-1)}$.

$$
\begin{aligned}
\tilde{\lambda}_{i}^{\{z\}} & =-\int_{-1}^{1} f(x)^{(z-1)}\left[u_{\gamma}(x) w(x) P_{i}(x)\right]^{(1)} \mathrm{d} x \\
& =-\left[f(x)^{(z-2)}\left[u_{\gamma}(x) w(x) P_{i}(x)\right]^{(1)}\right]_{-1}^{1}+\int_{-1}^{1} f(x)^{(z-2)}\left[u_{\gamma}(x) w(x) P_{i}(x)\right]^{(2)} \mathrm{d} x \\
& =\int_{-1}^{1} f(x)^{(z-2)}\left[u_{\gamma}(x) w(x) P_{i}(x)\right]^{(2)} \mathrm{d} x
\end{aligned}
$$

This is true if $u_{\gamma}^{(1)}(1)=u_{\gamma}^{(1)}(-1)=0$ and $f(1)^{(z-2)}, f(-1)^{(z-2)}, w^{(1)}(1), w^{(1)}(-1), P_{i}^{(1)}(1)$ and $P_{i}^{(1)}(-1)$ finite. This assumption is true for $w^{(1)}$ and $P_{i}^{(1)}$ and most of the time true for $f^{(z-2)}$.

And so on until (7):

$$
\tilde{\lambda}_{i}^{\{z\}}=(-1)^{z} \int_{-1}^{1} f(x)\left[u_{\gamma}(x) w(x) P_{i}(x)\right]^{(z)} \mathrm{d} x
$$

\section{Appendix B. $\alpha_{i+k}^{(\gamma, z)}$ coefficients for derivative computation}

Appendix B.1. $\alpha_{i+k}^{(\gamma, 1)}$ coefficients for first order derivative

For $i \geq 1$ :

$$
\begin{aligned}
\alpha_{i-1}^{(\gamma, 1)} & =-\left(\frac{1}{2}-\gamma+\frac{i}{2}\right) \\
\alpha_{i+1}^{(\gamma, 1)} & =-\left(\frac{1}{2}-\gamma-\frac{i}{2}\right) \\
\alpha_{i}^{(\gamma, 1)} & =0
\end{aligned}
$$

For $i=0$ :

$$
\begin{aligned}
& \alpha_{1}^{(\gamma, 1)}=-2\left(\frac{1}{2}-\gamma\right) \\
& \alpha_{-1}^{(\gamma, 1)}=\alpha_{0}^{(\gamma, 1)}=0
\end{aligned}
$$


Appendix B.2. $\alpha_{i+k}^{(\gamma, 2)}$ coefficients for second order derivative

For $i \geq 2$ :

$$
\begin{aligned}
\alpha_{i-2}^{(\gamma, 2)} & =\left(\frac{1}{2}-\gamma+i / 2\right)\left(1-\gamma+\frac{i}{2}\right) \\
\alpha_{i+2}^{(\gamma, 2)} & =\left(\frac{1}{2}-\gamma-i / 2\right)\left(1-\gamma-\frac{i}{2}\right) \\
\alpha_{i-1}^{(\gamma, 2)} & =\alpha_{i+1}^{(\gamma, 2)}=0 \\
\alpha_{i}^{(\gamma, 2)} & =\left((2 \gamma-1)(\gamma-1)-\frac{i^{2}}{2}\right)
\end{aligned}
$$

For $i=1$ :

$$
\begin{aligned}
& \alpha_{1}^{(\gamma, 2)}=\left[\left(\gamma-\frac{3}{2}\right)(-3 \gamma-2)-\gamma\right] \\
& \alpha_{3}^{(\gamma, 2)}=\gamma\left(\gamma-\frac{1}{2}\right) \\
& \alpha_{-1}^{(\gamma, 2)}=\alpha_{0}^{(\gamma, 2)}=\alpha_{2}^{(\gamma, 2)}=0
\end{aligned}
$$

For $i=0$ :

$$
\begin{aligned}
\alpha_{0}^{(\gamma, 2)} & =2\left(\gamma-\frac{1}{2}\right)(\gamma-2) \\
\alpha_{2}^{(\gamma, 2)} & =2\left(\gamma-\frac{1}{2}\right)(\gamma-1) \\
\alpha_{-2}^{(\gamma, 2)} & =\alpha_{-1}^{(\gamma, 2)}=\alpha_{1}^{(\gamma, 2)}=0
\end{aligned}
$$

Appendix B.3. $\alpha_{i+k}^{(\gamma, 3)}$ coefficients for third order derivative

For $i \geq 3$ :

$$
\begin{aligned}
& \alpha_{i-3}^{(\gamma, 3)}=\alpha_{i-2}^{(\gamma, 2)}\left(\frac{3}{2}-\gamma+\frac{i}{2}\right) \\
& \alpha_{i+3}^{(\gamma, 3)}=\alpha_{i+2}^{(\gamma, 2)}\left(\frac{3}{2}-\gamma-\frac{i}{2}\right) \\
& \alpha_{i-1}^{(\gamma, 3)}=\alpha_{i-2}^{(\gamma, 2)}\left(\frac{7}{2}-\gamma-\frac{i}{2}\right)+\alpha_{i}^{(\gamma, 2)}\left(\frac{5}{2}-\gamma+\frac{i}{2}\right) \\
& \alpha_{i+1}^{(\gamma, 3)}=\alpha_{i}^{(\gamma, 2)}\left(\frac{5}{2}-\gamma-\frac{i}{2}\right)+\alpha_{i+2}^{(\gamma, 2)}\left(\frac{7}{2}-\gamma+\frac{i}{2}\right) \\
& \alpha_{i-2}^{(\gamma, 3)}=\alpha_{i+2}^{(\gamma, 3)}=\alpha_{i}^{(\gamma, 3)}=0
\end{aligned}
$$


For $i=1$ :

$$
\begin{aligned}
& \alpha_{0}^{(\gamma, 3)}=\left[\left(\gamma-\frac{3}{2}\right)(-3 \gamma+2)+\gamma\right](3-\gamma) \\
& \alpha_{2}^{(\gamma, 3)}=\left[\left(\left(\gamma-\frac{3}{2}\right)(-3 \gamma+2)+\gamma\right)(2-\gamma)-\gamma\left(\gamma-\frac{1}{2}\right)(4-\gamma)\right] \\
& \alpha_{4}^{(\gamma, 3)}=-\gamma\left(\gamma-\frac{1}{2}\right)(1-\gamma) \\
& \alpha_{-2}^{(\gamma, 3)}=\alpha_{-1}^{(\gamma, 3)}=\alpha_{1}^{(\gamma, 3)}=\alpha_{3}^{(\gamma, 2)}=0
\end{aligned}
$$

For $i=0$ :

$$
\begin{aligned}
& \alpha_{0}^{(\gamma, 3)}=2\left(\gamma-\frac{1}{2}\right)\left[\left(\left(\gamma-\frac{5}{2}\right)(3 \gamma-5)-(\gamma-1)\right)\right] \\
& \alpha_{3}^{(\gamma, 3)}=2\left(\gamma-\frac{1}{2}\right)\left(\gamma-\frac{3}{2}\right)(\gamma-1) \\
& \alpha_{-3}^{(\gamma, 3)}=\alpha_{-2}^{(\gamma, 3)}=\alpha_{-1}^{(\gamma, 3)}=\alpha_{1}^{(\gamma, 3)}=\alpha_{2}^{(\gamma, 3)}=0
\end{aligned}
$$

For $i=2$, the combination process is similar to other coefficient orders. Appendix B.4. $\alpha_{i+k}^{(\gamma, 4)}$ coefficients for the fourth order derivative

For $i \geq 4$ :

$$
\begin{aligned}
& \alpha_{i-4}^{(\gamma, 4)}=\alpha_{i-3}^{(\gamma, 3)}\left(2-\gamma+\frac{i}{2}\right) \\
& \alpha_{i+4}^{(\gamma, 4)}=\alpha_{i+3}^{(\gamma, 3)}\left(2-\gamma-\frac{i}{2}\right) \\
& \alpha_{i-2}^{(\gamma, 4)}=\alpha_{i-3}^{(\gamma, 3)}\left(5-\gamma-\frac{i}{2}\right)+\alpha_{i-1}^{(\gamma, 3)}\left(3-\gamma+\frac{i}{2}\right) \\
& \alpha_{i+2}^{(\gamma, 4)}=\alpha_{i+1}^{(\gamma, 3)}\left(3-\gamma-\frac{i}{2}\right)+\alpha_{i+3}^{(\gamma, 3)}\left(5-\gamma+\frac{i}{2}\right) \\
& \alpha_{i}^{(\gamma, 4)}=\alpha_{i-1}^{(\gamma, 3)}\left(4-\gamma-\frac{i}{2}\right)+\alpha_{i+1}^{(\gamma, 3)}\left(4-\gamma+\frac{i}{2}\right) \\
& \alpha_{i-3}^{(\gamma, 4)}=\alpha_{i+3}^{(\gamma, 4)}=\alpha_{i-1}^{(\gamma, 4)}=\alpha_{i+1}^{(\gamma, 4)}=0
\end{aligned}
$$

For $i=0,1,2,3$, the combination process is similar to other coefficient orders.

\section{Bibliography}

\section{References}

[1] H. Garnier, L. Wang, Identification of continuous-time models from sampled data, Springer Verlag, 2008. 
[2] D. Remond, J. Neyrand, G. Aridon, R. Dufour, On the improved use of Chebyshev expansion for mechanical system identification, Mechanical Systems and Signal Processing 22 (2) (2008) 390 - 407, ISSN 0888-3270.

[3] G. de Melo, T. Morais, Fault detection using state observers with unknown input, identified by orthogonal functions and PI observers, Brazilian Society of mechanical sciences and Engineering .

[4] L. Ljung, A. Wills, Issues in sampling and estimating continuous-time models with stochastic disturbances, Automatica 46 (5) (2010) 925 - 931, ISSN 0005-1098, doi: DOI: $10.1016 /$ j.automatica.2010.02.011.

[5] R. Pacheco, V. Steffen, Using orthogonal functions for identification and sensitivity analysis of mechanical systems, Journal of Vibration and Control 8 (7) (2002) 9931021.

[6] R. Pacheco, V. Steffen Jr, Orthogonal function techniques for the identification of nonlinear mechanical systems, in: Materials science forum, vol. 440, 59-68, 2003.

[7] Q. Leclère, C. Pézerat, Vibration source identification using corrected finite difference schemes, Journal of Sound and Vibration 331 (6) (2012) 1366 - 1377, ISSN 0022-460X, doi:10.1016/j.jsv.2011.11.002, URL http://www.sciencedirect.com/science/article/pii/S0022460X11008807.

[8] C. Pezerat, J. Guyader, Two inverse methods for localization of external sources exciting a beam, Acta Acustica 3 (1) (1995) 1-10.

[9] S. Chesne, C. Pezerat, Distributed piezoelectric sensors for boundary force measurements in Euler-Bernoulli beams, Smart Materials and Structures 20 (2011) 075009.

[10] S. Chesne, C. Pezerat, J. Guyader, Identification of Plate Boundary Forces From Measured Displacements, Journal of Vibration and Acoustics 130 (2008) 041006.

[11] M. Mboup, C. Join, M. Fliess, Numerical differentiation with annihilators in noisy environment, Numerical Algorithms 50 (4) (2009) 439-467, doi:10.1007/s11075-0089236-1.

[12] D. Wu, S. Law, Damage localization in plate structures from uniform load surface curvature, Journal of Sound and Vibration 276 (1-2) (2004) 227-244.

[13] J. Mason, D. Handscomb, Chebyshev polynomials, Chapman \& Hall/CRC, ISBN $9780849303555,2003$.

[14] L. D-Y., O. Gibaru, W. Perruquetti, Error analysis of Jacobi derivative estimators for noisy signals, Numerical Algorithms 58 (2011) 53-83, ISSN 1017-1398, $10.1007 / \mathrm{s} 11075-011-9447-8$. 


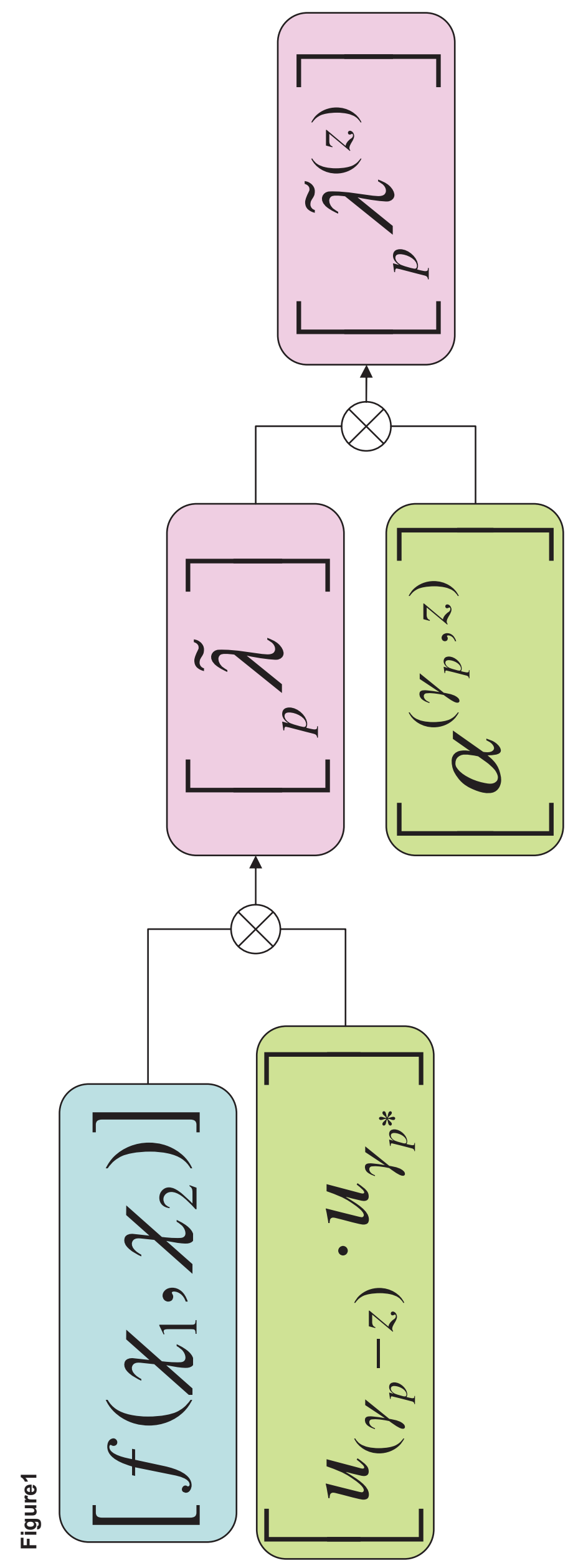




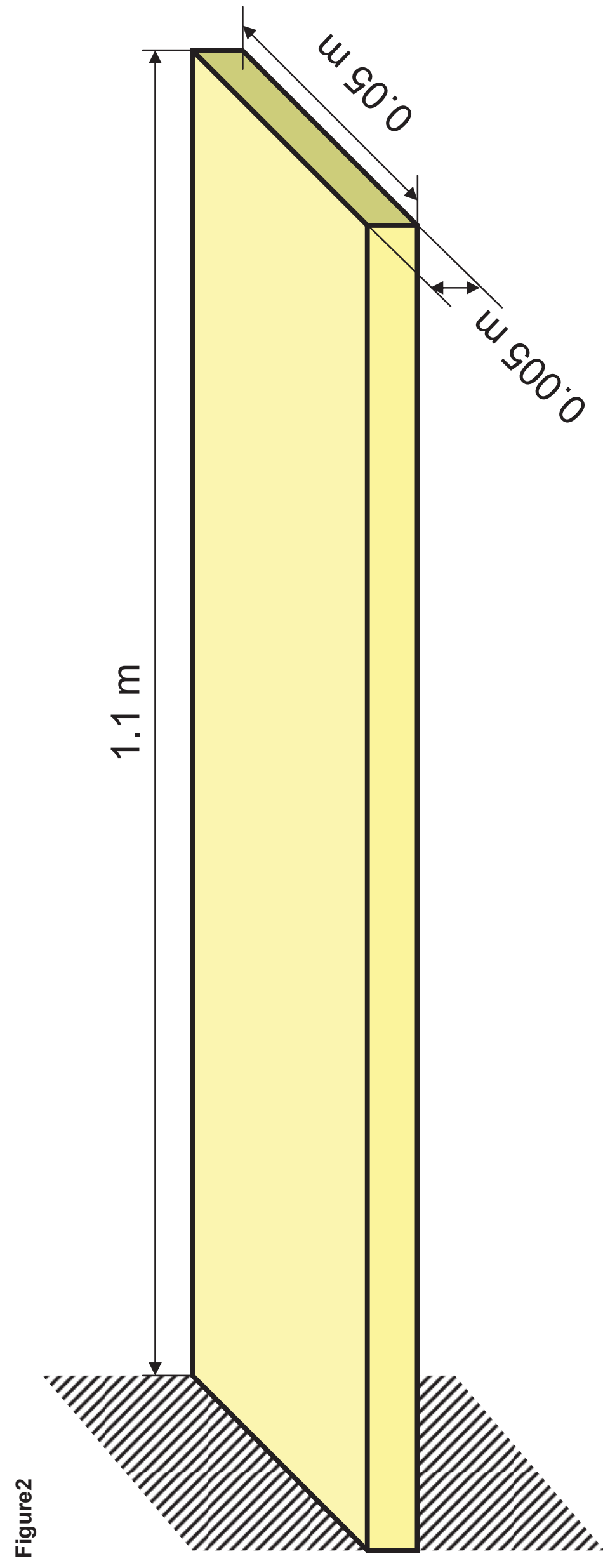



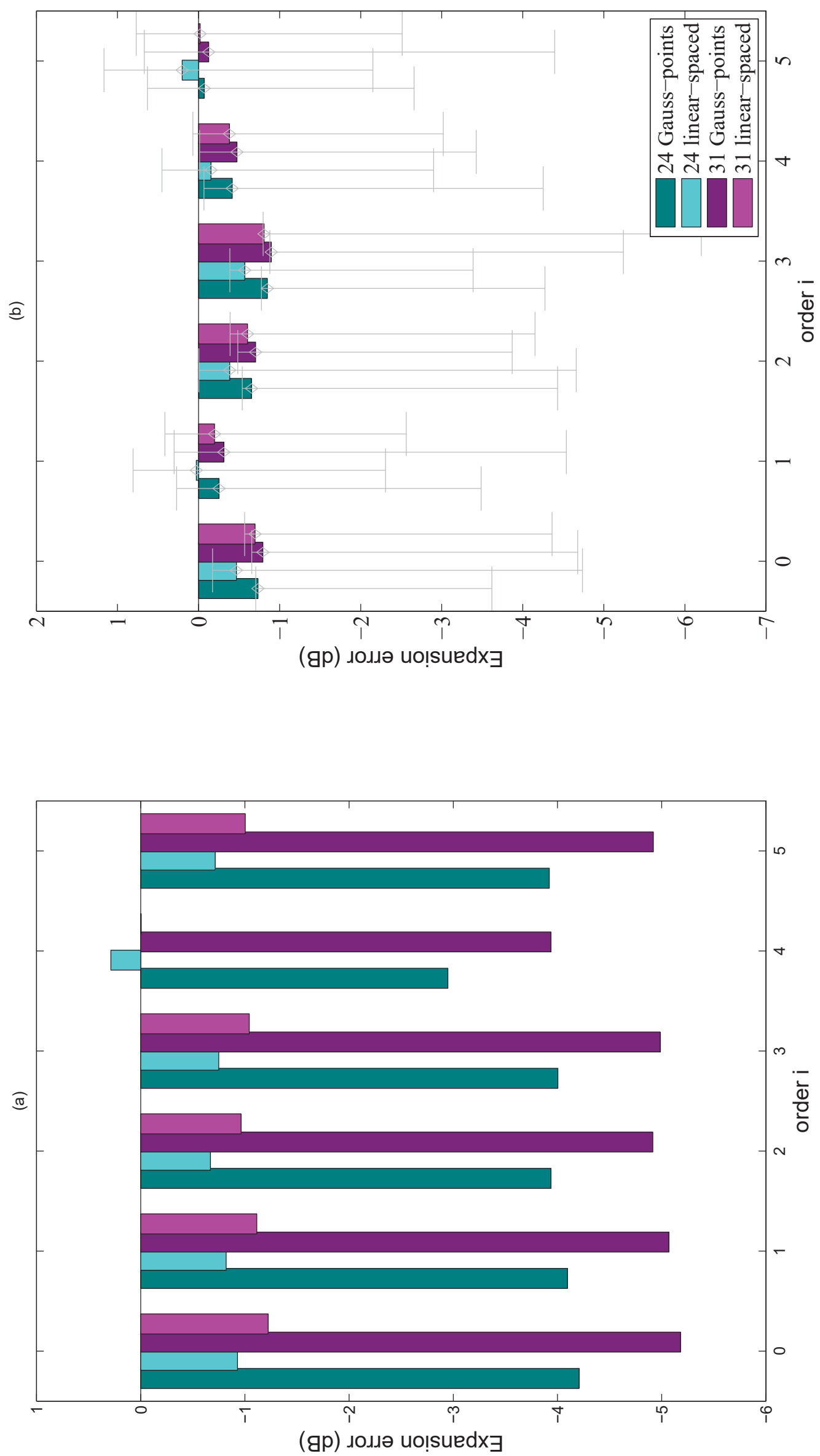

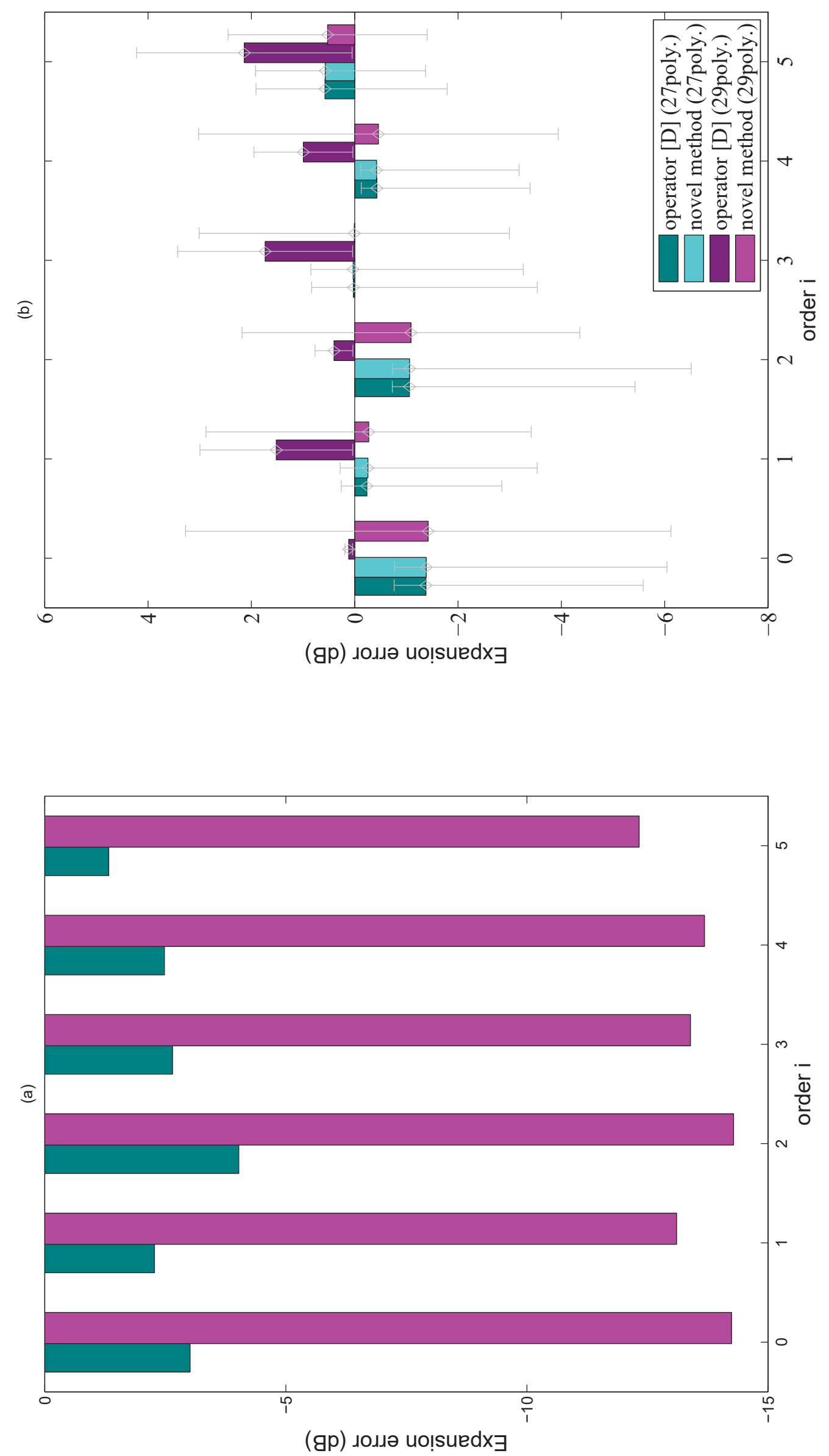


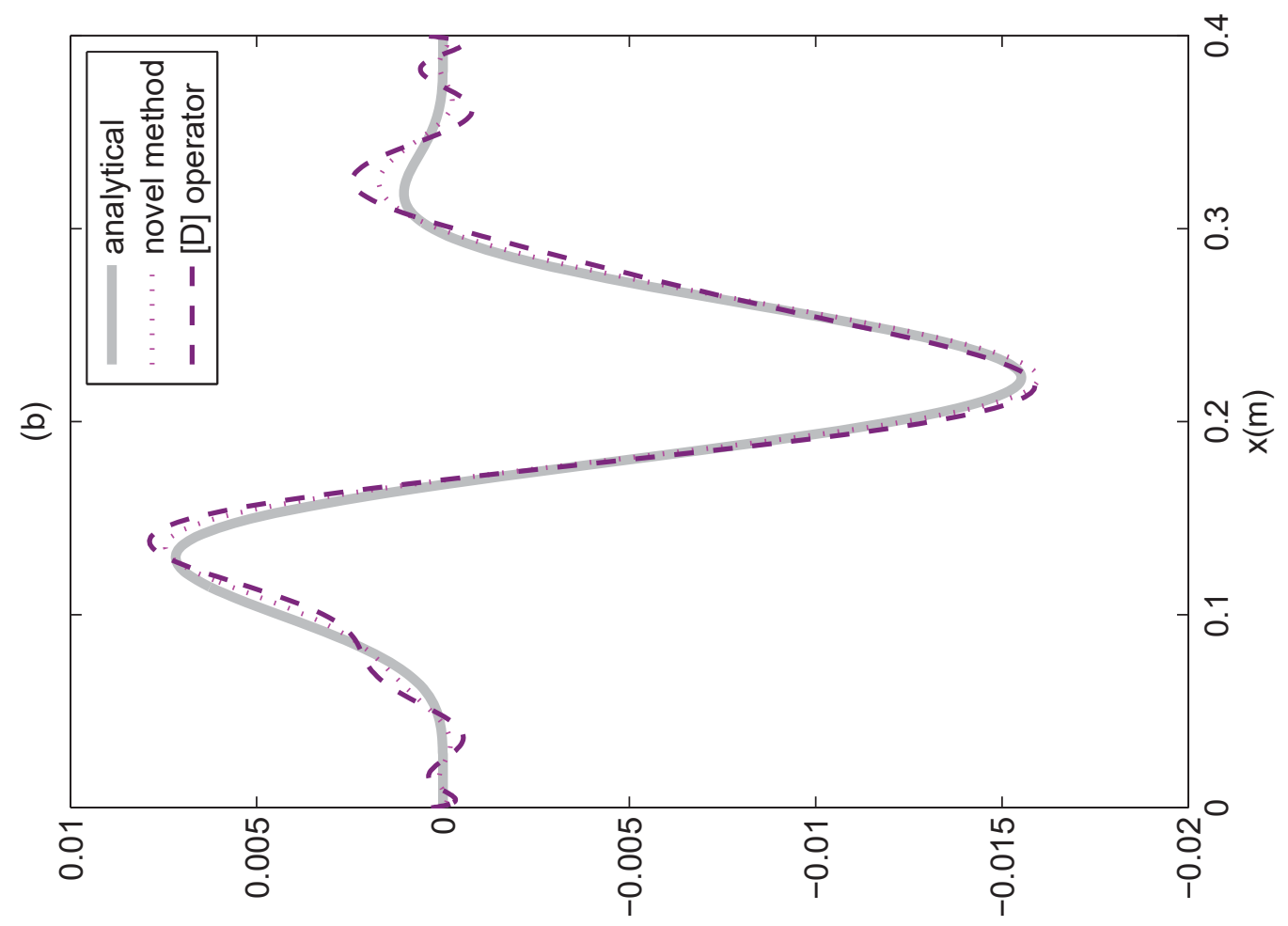

$(x) n \cdot(x)_{(t)} \wedge$

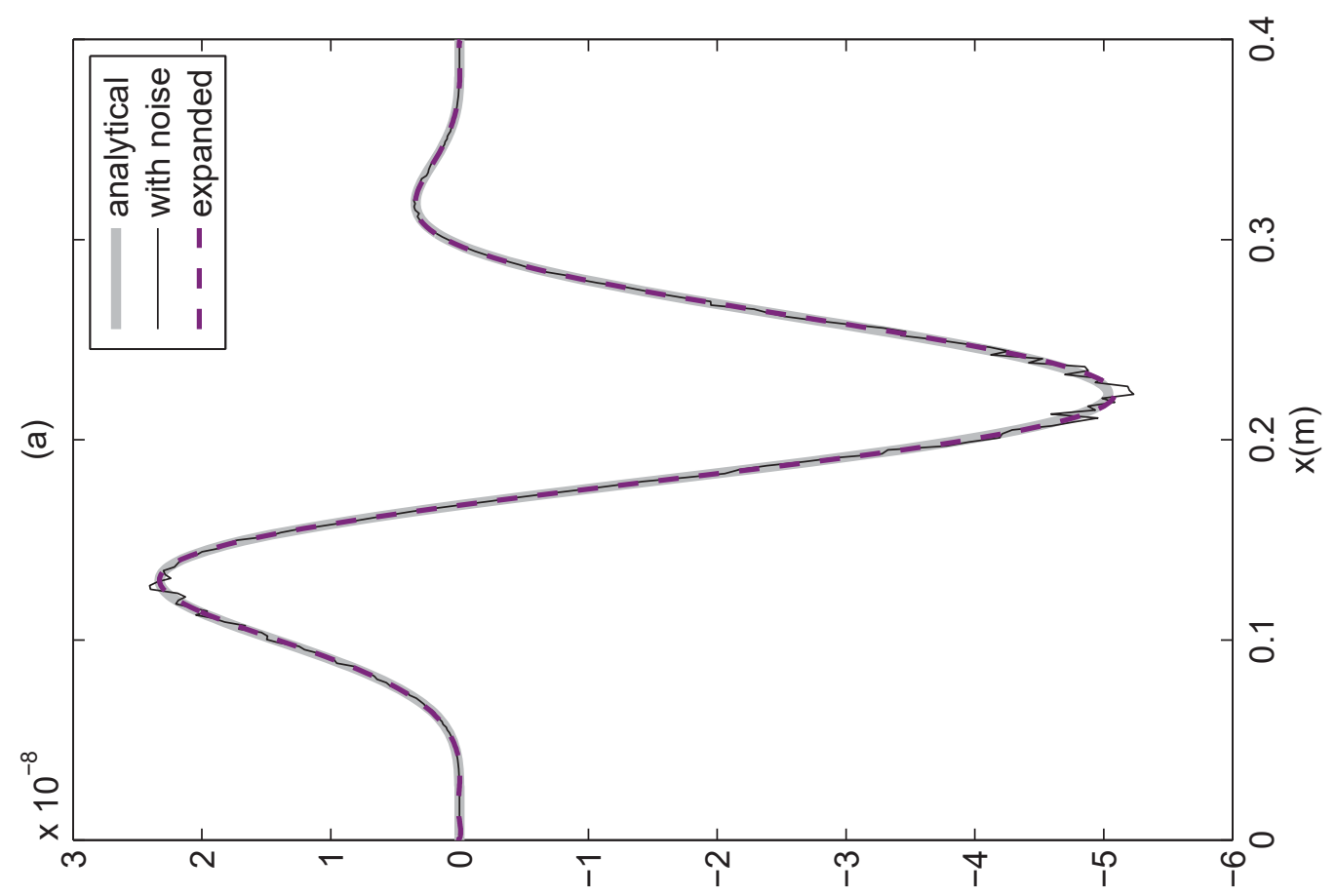

$(x) n \cdot(x) \wedge$

ํํㄴ 

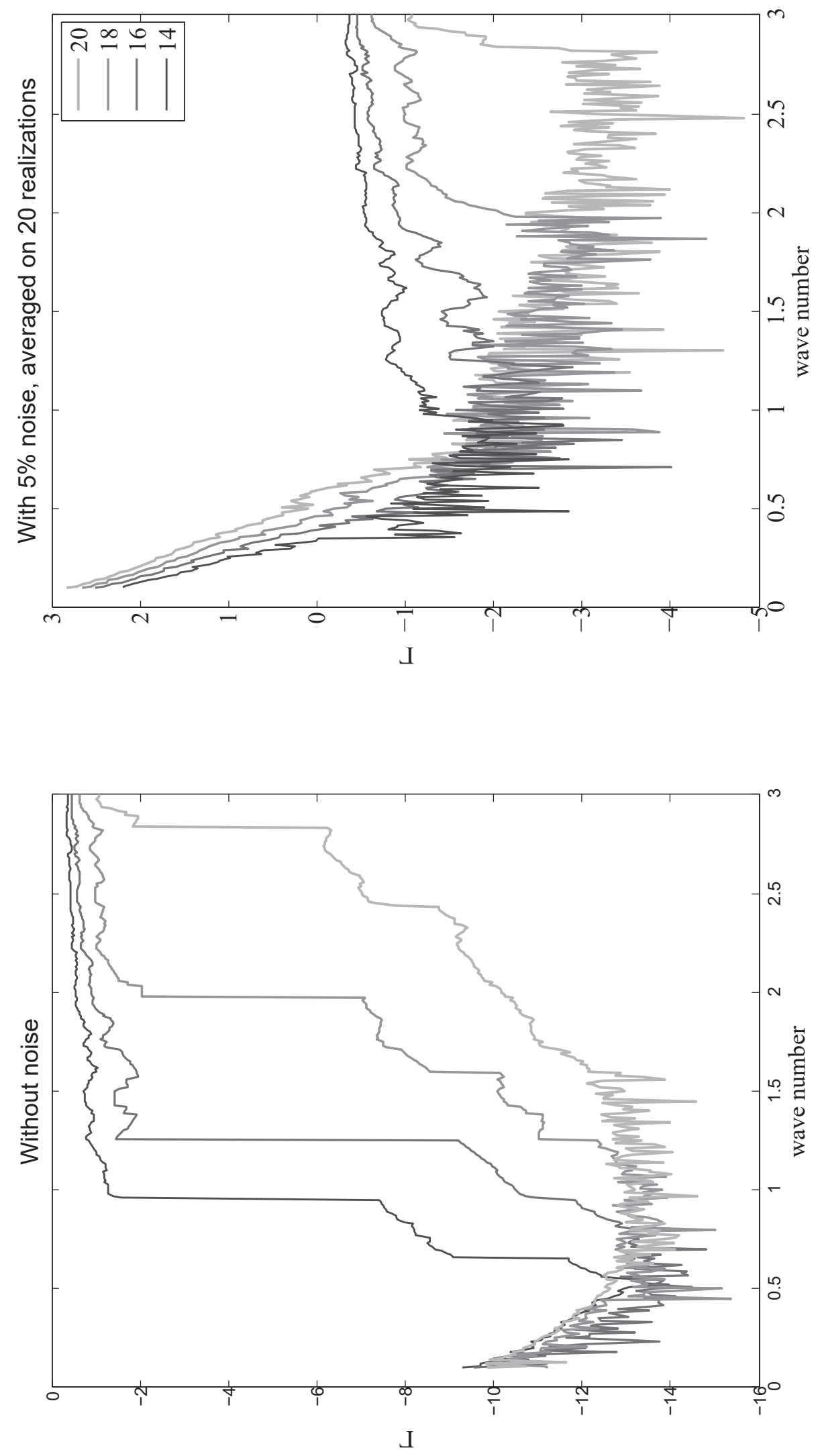


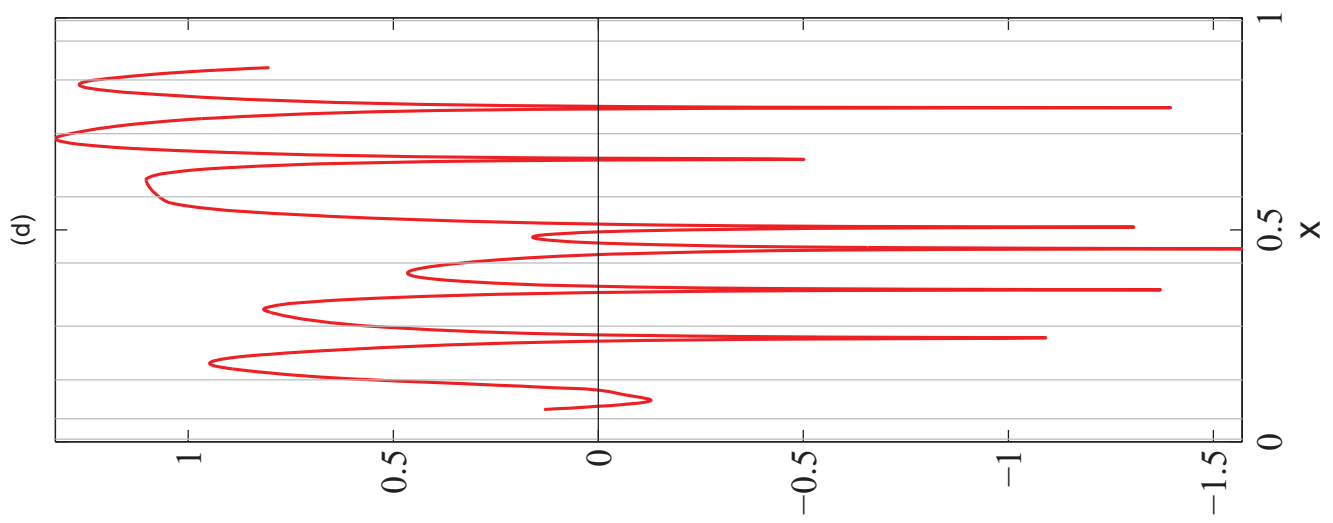

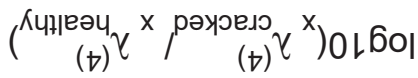

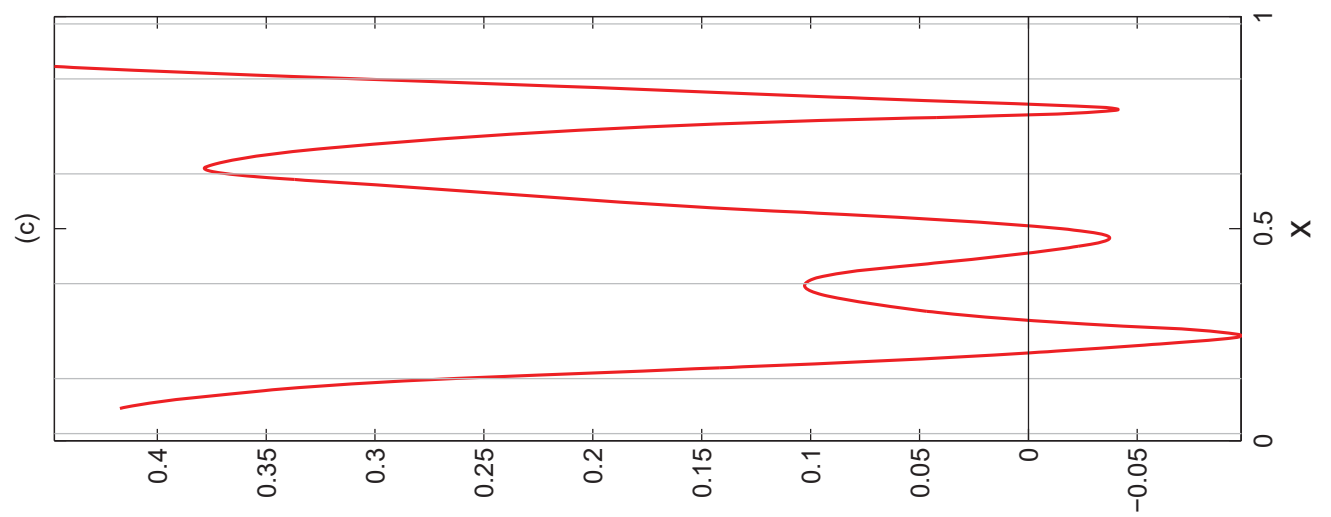

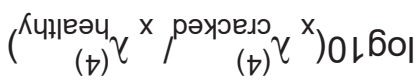
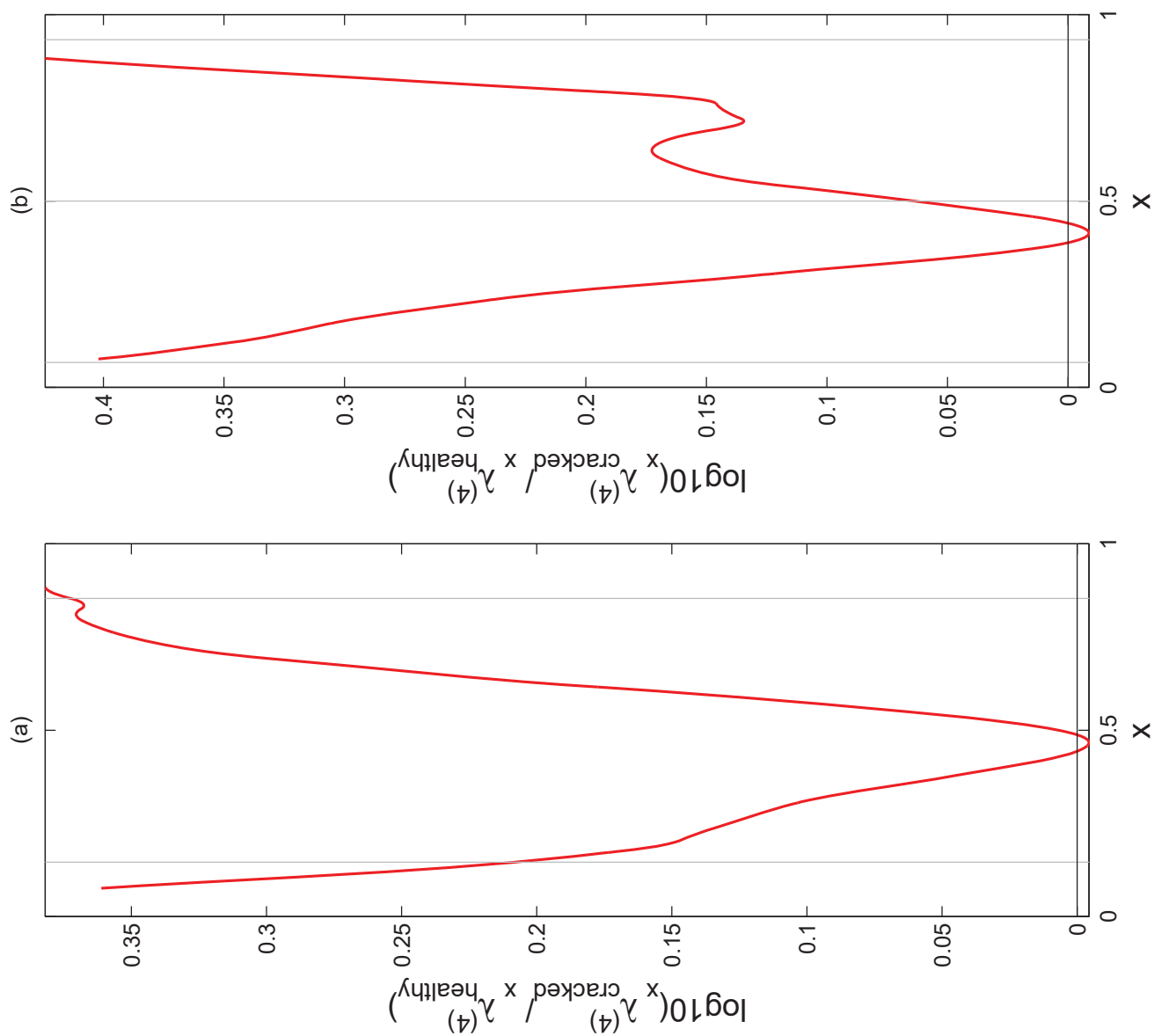


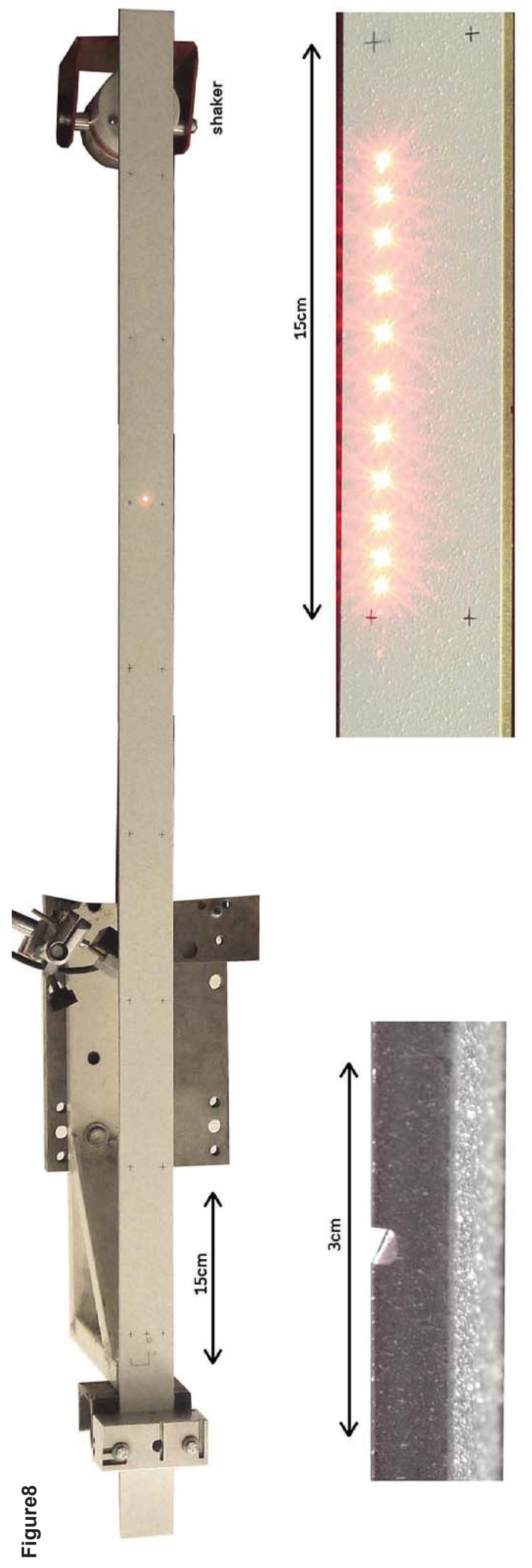




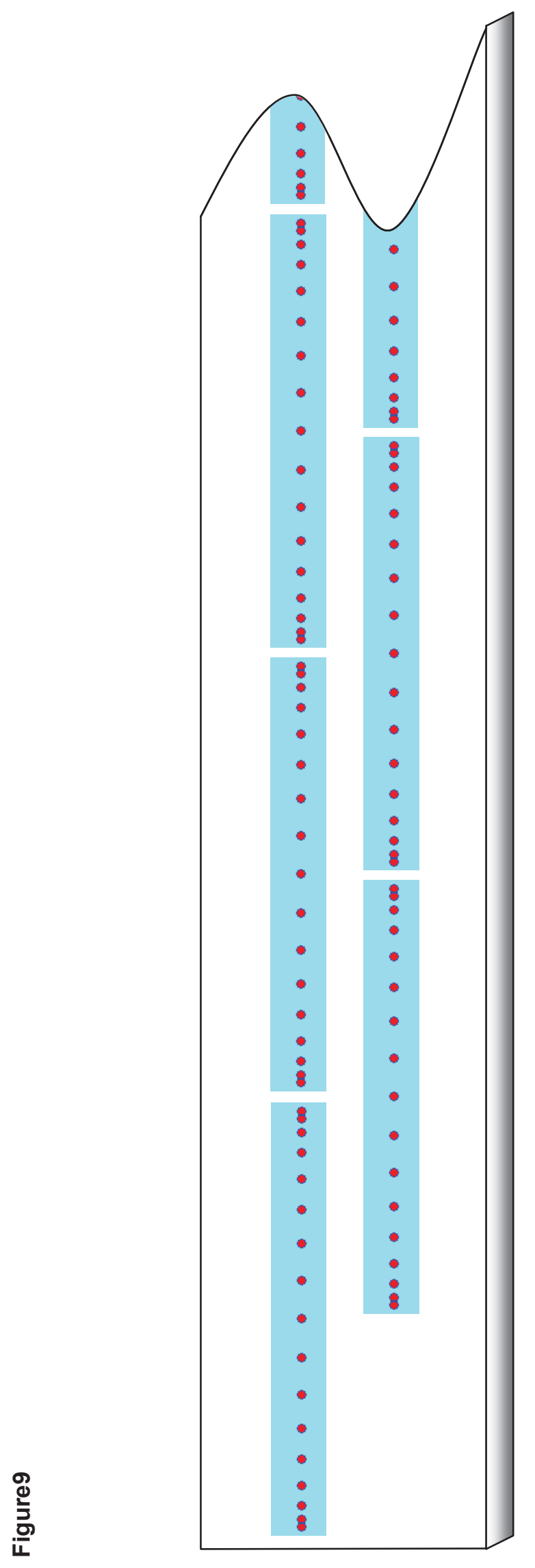



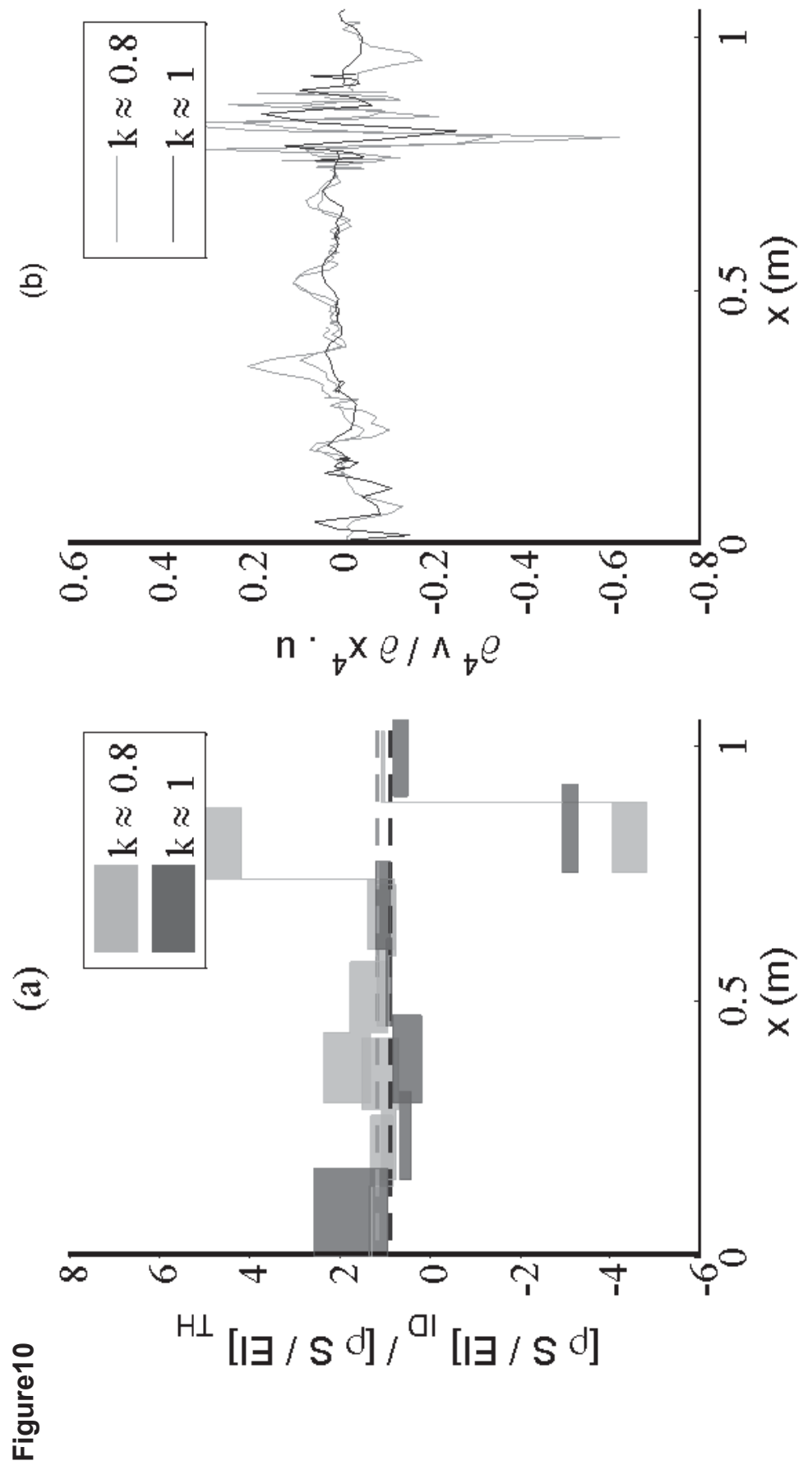